\title{
Unique properties of $\mathrm{RV}$ in an in vitro model of asthma exacerbation
}

\author{
Ramses llarraza*, Yingqi Wu, Darryl Adamko \\ From AllerGen NCE Inc.'s Fifth Annual Research Conference: Innovation from Cell to Society \\ Québec City, QC, Canada. 7-9 February 2010
}

\section{Background}

A vast majority of asthma exacerbations are linked to viral infections, most of which are caused by rhinovirus (RV). We hypothesize that eosinophils are key effector cells in asthma exacerbations. As such, using an in vitro model of human cells, we have shown that respiratory syncytial virus (RSV) and RV induce eosinophil degranulation when co-cultured with $\mathrm{T}$-cells and autologous monocyte-derived dendritic cells (moDC), concurrent with moDC-dependent CD4+ CD45RO+ T-cell activation. While our original hypothesis has been that such activation is mediated solely by antigen-presentation (AP), our recent data also suggest that RV may be the major pathogen associated with asthma attacks because of its unique ability to interact directly with T-cells.

\section{Materials and methods}

Human monocytes, T-lymphocytes, autologous moDC and eosinophils (EOS) were isolated from blood. moDC were cultured with RSV, RV, or sham, then washed, and incubated with autologous T-cells. To determine the role for antigen presentation, some moDC were treated with the inhibitor chloroquine before virus culture. In some, no moDc were added and T lymphocytes (CD4, CD8 or a mix) were exposed to RSV, RV or UV-inactivated RV (RV-UV). T-cell activation and apoptosis were measured by flow cytometry (CD25 expression and Annexin-V/TO-PRO-3 staining, respectively), and proliferation by BrdU incorporation (Cell Proliferation ELISA, Roche). EOS cysteinyl-leukotriene (Cys-LT) release was measured by ELISA (Cayman Chemical). Cytokine release was measured by Searchlight (Pierce).

\section{Results}

RV presence induced a 5-fold increase in Cys-LT release from EOS co-cultured with T-cells and moDC, while RSV did not have any effect. In T-cell and moDC cocultures, RV induced greater increases in IL-1beta, IL10, IL-12p70, IL-13 and IFN-gamma compared with RSV. T-cells, and more evidently CD8+ T-cells, showed increased apoptosis when incubated with RV but not with RV-UV or RSV. Virus-loaded moDC induced proliferation of T-cells, but, unlike RSV, RV was insensitive to chloroquine.

\section{Conclusions}

Our results suggest that RSV and RV induce EOS and T-cell activation differentially. RSV induces proliferation in a MHC-II restricted antigen-presentation-dependent manner, while for RV this is not the sole pathway. In addition, based on cytokine and Cys-LT release, RV appears to be more potent compared to RSV. Our data are relevant to help understand why RV is so potent in the induction of asthma exacerbation in allergic patients, and will lead to novel potential targets for improved preventative strategies.

Published: 26 November 2010

doi:10.1186/1710-1492-6-S3-P17

Cite this article as: llarraza et al:: Unique properties of RV in an in vitro model of asthma exacerbation. Allergy, Asthma \& Clinical Immunology 2010 6(Suppl 3):P17. 